\title{
Erratum
}

Phil. Trans. R. Soc. Lond. B 353, 187-198 (February 1998)

\section{Levels of genetic polymorphism: marker loci versus quantitative traits}

\section{R. K. Butlin and T. Tregenza}

Table 1 on p. 193 of this paper includes some erroneous values due to a mistake in a spreadsheet that has been discovered through a re-analysis of the data. The errors do not affect the conclusions drawn in the paper. A correct version of the table follows.

Table 1. $\chi^{2}$ isolation indices between pairs of European populations of the meadow grasshopper

(Locations of populations are given in figure 2.)

\begin{tabular}{lrccccc}
\hline population & 1 & 2 & 3 & 4 & 5 & 6 \\
\hline 1. S. France & & & & & & \\
2. S. France & 0.067 & & & & & \\
3. N. France & -0.122 & $0.500^{*}$ & & & & \\
4. England & 0.198 & -0.063 & $0.472^{*}$ & & & \\
5. Spain & -0.088 & $0.545^{*}$ & -0.365 & 0.272 & & \\
6. Germany & 0.056 & 0.113 & 0.189 & 0.230 & 0.045 & \\
7. N. Greece & 0.380 & $0.381^{*}$ & $0.527^{*}$ & $0.306^{*}$ & $0.547^{*}$ & $0.499^{*}$ \\
\hline
\end{tabular}

*Indicates that the individual isolation index is significantly different from 0 (i.e. departs from random mating) at $p<0.05$. Positive indices indicate assortative mating. 